\title{
Effect of positive end-expiratory pressure on porcine right ventricle function assessed by speckle tracking echocardiography
}

\author{
Sam R Orde ${ }^{1,2^{*}}$, Atta Behfar ${ }^{1}$, Paul G Stalboerger ${ }^{1}$, Sergio Barros-Gomes ${ }^{1}$, Garvan C Kane ${ }^{1}$ and Jae K Oh ${ }^{1}$
}

\begin{abstract}
Background: Right ventricle (RV) dysfunction and hypotension can be induced by high levels of positive end-expiratory pressure (PEEP). We sought to determine in an animal model if a novel ultrasound analysis technique: speckle tracking echocardiography (STE), could determine deterioration in RV function induced by PEEP and to compare this to a conventional method of RV analysis: fractional area change (FAC). STE is a sensitive, angle-independent method for describing cardiac deformation ('strain') and is particularly useful in analyzing RV function as has been shown in pulmonary hypertension cohorts.
\end{abstract}

Methods: Ten pigs, 40-90 kg, anaesthetized, fully mechanically ventilated at $6 \mathrm{ml} / \mathrm{kg}$ were subject to step-wise escalating levels of PEEP at two-minute intervals $\left(0,5,10,15,20,25\right.$ and $\left.30 \mathrm{cmH}_{2} 0\right)$. Intracardiac echocardiography was used to image the RV as transthoracic and transesophageal echocardiography did not give sufficient image quality or flexibility. Off-line STE analysis was performed using Syngo Velocity Vector Imaging (Seimens Medical Solutions Inc., USA). STE systolic parameters are RV free wall strain (RVfwS) and strain rate (RVfwSR) and the diastolic parameter RV free wall strain rate early relaxation (RVfwSRe)

Results: With escalating levels of PEEP there was a clear trend of reduction in STE parameters (RVfwS, RVfwSR, RVfwSRe) and FAC. Significant hypotension (fall in mean arterial blood pressure of $20 \mathrm{mmHg}$ ) occurred at approximately PEEP $15 \mathrm{cmH}_{2} \mathrm{O}$. Comparing RVfwS, RVfwSR and RVfwSRe values at different PEEP levels showed a significant difference at PEEP $0 \mathrm{cmH}_{2} \mathrm{O}$ vs PEEP $10 \mathrm{cmH}_{2} \mathrm{O}$ and above. FAC only showed a significant difference at PEEP $0 \mathrm{cmH}_{2} \mathrm{O}$ vs PEEP 20 $\mathrm{CmH}_{2} \mathrm{O}$ and above. $30 \%$ of pigs displayed dyssychronous RV free wall contraction at the highest PEEP level reached.

Conclusions: STE is a sensitive method for determining RV dysfunction induced by PEEP and deteriorated ahead of a conventional assessment method: FAC. RVfwS decreased to greater extent compared to baseline than FAC, earlier in the PEEP escalation process and showed a significant decrease before there was a clinical relevant decrease in mean arterial blood pressure. Studies in ICU patients using transthoracic echocardiography are warranted to further investigate the most sensitive echocardiography method for detecting RV dysfunction induced by mechanical ventilation.

Keywords: Speckle tracking echocardiography, Right ventricle, Right ventricle strain, PEEP, Mechanical ventilation

\section{Background}

Right ventricle (RV) failure in the critically ill is an independent risk factor for mortality in patients with acute lung injury and acute respiratory distress syndrome (ARDS) [1,2]. It can be challenging to treat and requires accurate and early recognition in order to tailor treatment

\footnotetext{
* Correspondence: samorde@hotmail.com

'Division of Cardiovascular Diseases, Mayo Clinic, 200 First St SW, Rochester, MN 55905, USA

${ }^{2}$ Intensive Care Unit, Nepean Hospital, Kingswood, Sydney 2749, NSW, Australia
}

\section{Biomed Central}

$[3,4]$. Echocardiography has a crucial role in the diagnosis of RV failure in the ICU [5]. Interpretation can be difficult however, due to the crescentric shape, retrosternal position and the poor correlation between conventional assessment methods, such as fractional area change (FAC) and intrinsic RV contractile dysfunction [6] as well as translational errors with methods such as tricuspid annular plane systolic excursion and tissue Doppler imaging. Speckle tracking echocardiography (STE) has emerged as a relatively novel, angle-independent technique for 
analyzing the grey-scale ultrasound (B mode) images of the heart [7] and can elucidate cardiac dysfunction not seen with conventional echocardiography techniques $[8,9]$. STE is particularly useful for assessing RV systolic function: $\mathrm{RV}$ free wall strain (RVfwS) and RV free wall strain rate (RVfwSR) which are suggested to be more robust measures of RV contractility than conventional echocardiography methods in diseases such as pulmonary hypertension [10-13].

Positive end expiratory pressure (PEEP) is an integral component of mechanical ventilation in critically ill patients suffering from acute lung injury and ARDS, yet can have negative consequences on cardiac haemodynamics [14]. 'Open-lung ventilation' aims to decrease the cyclic opening and closing of small distal airways and atelectatic alveoli which can lead to ventilator-induced lung injury $[15,16]$ through the use of elevated PEEP levels. Cardiac function can be affected by high PEEP levels in several ways including: biventricular reduced venous return and increased right ventricle (RV) afterload, which is poorly tolerated [17] resulting in RV dysfunction, cor pulmonale and acute hypotension [3].

The aim of this study was to perform a step-wise PEEP escalation maneuver in anesthetized, fully mechanically ventilated pigs and to assess their RV function with STE and a conventional echocardiography measure of RV function analysis: FAC. We sought to 1) Determine if STE could describe changes in RV function induced by escalating levels of PEEP; 2) To compare RVfwS to a conventional measure of RV function assessment: FAC; and 3) Determine if RVfwS or FAC deterioration occurred prior to PEEP induced hypotension (defined as a fall in mean arterial blood pressure [MAP] of $20 \mathrm{mmHg}$ ).

\section{Methods}

All animal experiments and protocols were approved and carried out according to the guidelines of the Animal Care and Use Committee of the Mayo Clinic (Rochester, MN, USA). In 10 Yorkshire female swine weighing median $45 \mathrm{~kg}$ (41.5 to 60.5 interquartile range [IQR]), after overnight fasting, anesthesia was induced with Telazol $(5 \mathrm{mg} / \mathrm{kg}$ intramuscularly) and Xylazine $(2.0 \mathrm{mg} / \mathrm{kg}$ intramuscularly). The animals were intubated with a $7 \mathrm{~mm}$ internal diameter endotracheal tube, mechanically ventilated by a Datex-Ohmeda 7100 ventilator (GE Healthcare) with volume control mode at tidal volumes of $6 \mathrm{ml} / \mathrm{kg}$, fraction of inspired oxygen (FiO2) started at 0.4 aiming for saturations greater than $92 \%$, inspiratory/ expiratory ratio of $1: 2$, end-inspiratory pause of $10 \%$, respiratory rate of 16 breaths per minute and initial PEEP of $0 \mathrm{cmH}_{2} \mathrm{O}$. Anesthesia was maintained with inhaled isoflurane $1.0-3.0 \%$. The pigs were placed in a supine position during the entire experiment.
Percutaneous access was achieved through the femoral artery and both femoral veins for monitoring of arterial pressures ( $9 \mathrm{~F}$ sheath), pulmonary artery pressures (831HF75P, Swan-Ganz 7.5 French, Edwards Life-sciences, Irvine, $\mathrm{CA}$ ) and for the intracardiac echocardiography (ICE) catheter respectively. Correct positioning of the pulmonary artery catheter in the pulmonary artery was confirmed with fluoroscopy (see Figure 1), waveform analysis and by inflation of the catheter balloon. All intravascular catheters were zeroed to the atmosphere. The mid-point of the anterior and posterior chest was considered the reference point. Electrocardiogram and intravascular pressures were monitored continuously.

Due to the mediastinal anatomy of the swine, transthoracic and transoesophageal echocardiography did not provide sufficient image quality or flexibility to sufficiently image the RV free wall. We therefore had to use ICE, performed with an 8 French AcuNav ultrasound catheter connected to an Acuson SC2000 ultrasound machine (Siemens Medical USA, Malvern, Pennsylvania) inserted into the right atrium. Imaging was performed by A.B. (who is appropriately trained in this method) and was optimized for maximal frame rate to enable accurate speckle tracking and focused on the RV free wall in the long axis ensuring the tricuspid annulus was visible throughout the cardiac cycle (see Figure 2). The mean $( \pm$ SD) frame rate was $113( \pm 13)$ frames per second.

Once baseline stability was achieved, PEEP was increased in a stepwise manner every 2 minutes from PEEP 0 $\mathrm{cmH}_{2} \mathrm{O}$ to PEEP $30 \mathrm{cmH}_{2} \mathrm{O}$ in $5 \mathrm{cmH}_{2} \mathrm{O}$ increments keeping the tidal volumes constant (see Figure 3). Before

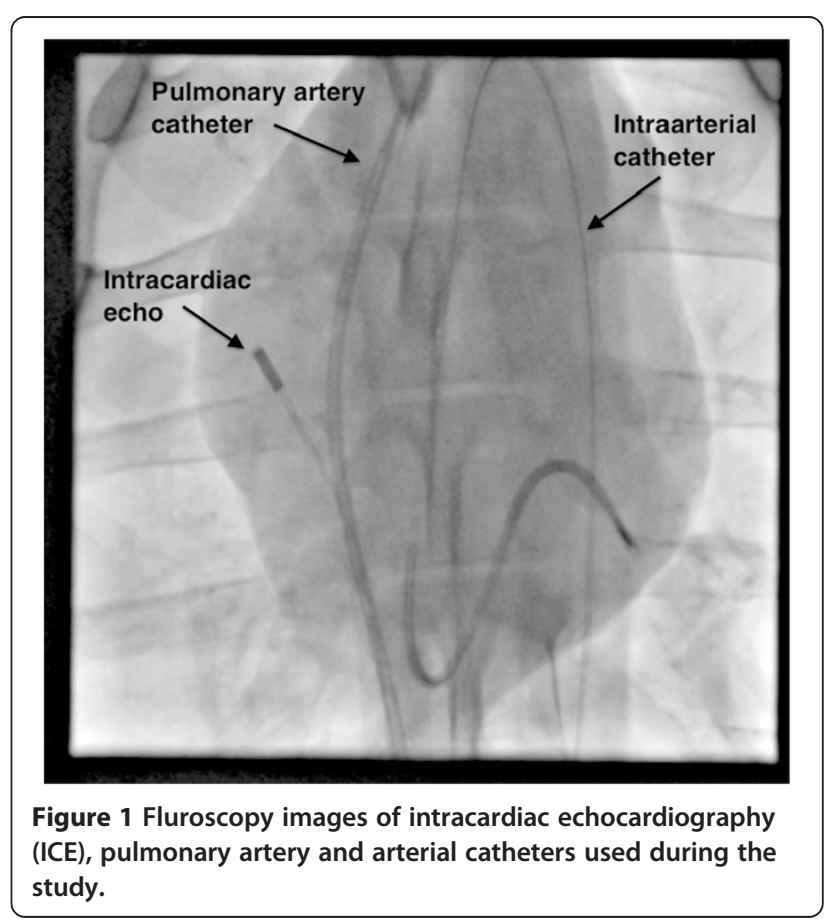



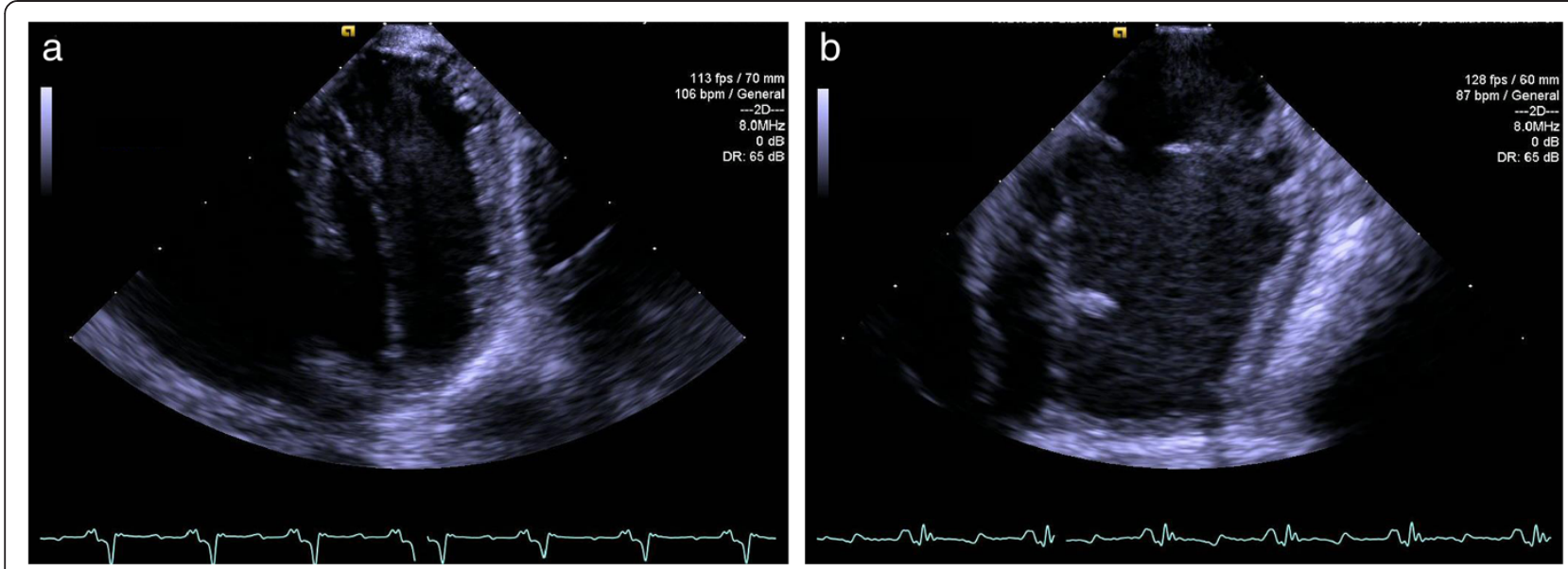

Figure 2 Representative intracardiac echocardiogram (ICE) images of the right ventricle (RV) at end-expiration and end-diastole. (a) PEEP 0 and (b) PEEP 30. Imaging optimized to assess the RV free wall, including the tricuspid annulus throughout the cardiac cycle, maximizing for frame rate to allow for accurate speckle tracking assessment.

each increase in PEEP, recordings were made at endexpiration: intracardiac echocardiography clips of 3 seconds, haemodynamic parameters and saturations. Significant hypotension was considered a decrease in MAP of $20 \mathrm{mmHg}$. The stepwise PEEP maneuver was ceased at PEEP $30 \mathrm{cmH}_{2} \mathrm{O}$ and PEEP returned to $0 \mathrm{cmH}_{2} \mathrm{O}$. The maneuver was ceased earlier if MAP fell below $25 \mathrm{mmHg}$, heart rate fell below 40 beats per minute or if oxygen saturation fell below $60 \%$ and was unresponsive to $\mathrm{FiO} 2$ of 1.0.

Two-dimensional ICE images were transferred to a Syngo Velocity Vector Imaging workstation (Siemens Medical USA, Malvern, Pennsylvania). A single best cardiac cycle was chosen to determine FAC by manually tracing the RV endocardium at end-diastole and end-systole: $\mathrm{FAC}=([$ end diastolic area - end systolic area $] /$ end-diastolic

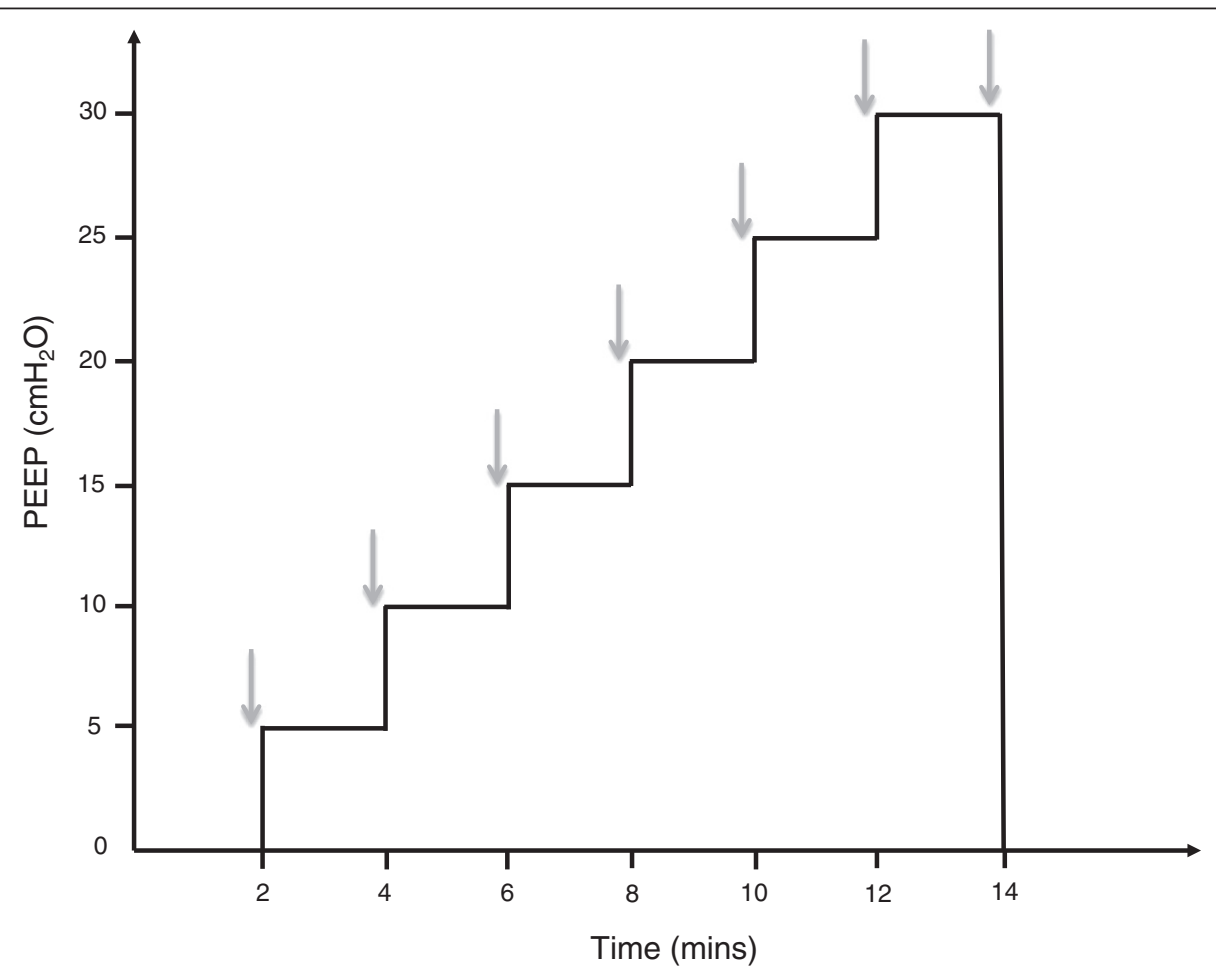

Figure 3 Graphical representation of the step-wise escalating PEEP protocol. Indicates timing of recordings made at end-expiration: physiological data and intracardiac echocardigraphy (ICE) images for post processing analysis. 
area) $x$ 100. One to three beat cardiac cycles were chosen for STE analysis. The endocardium was traced manually at end-systole from the medial to lateral annulus with approximately 7-15 points. RV function values are an average of the three free wall segments. Systolic function parameters are measures of deformation or strain: RVfwS and RVfwSR (change in strain/time) and these are negative values: the more negative the value the better the function. Diastolic function is determined by a positive value: RV free wall strain rate early relaxation (RVfwSRe): the speed that deformation returns to the end-diastolic value. Strain and strain rate curves were chosen based on appropriate tracking as well as assessing displacement, velocity, strain and strain rate curves for appropriate motion, smoothness and segment correlation. The same cardiac cycle was chosen for strain and strain rate values. See Figure 4 for examples of strain and strain rate curves at PEEP $0 \mathrm{cmH}_{2} \mathrm{O}$ and final PEEP value.

To assess for synchrony of RV free wall segment contraction we used a method proposed by Yu et al. [18] to assess for dyssynchrony of the left ventricle. The Time To Peak (TTP) strain value is determined by comparing the time taken from the onset of the QRS to peak strain value for each segment. TTP delay is the time difference between the segments with the smallest TTP compared to segment with the largest (see Figure 4: Maximal PEEP strain curve). The mean of the TTP delay at PEEP 0 $\mathrm{cmH}_{2} \mathrm{O}$ was used as the reference value and 2 standard

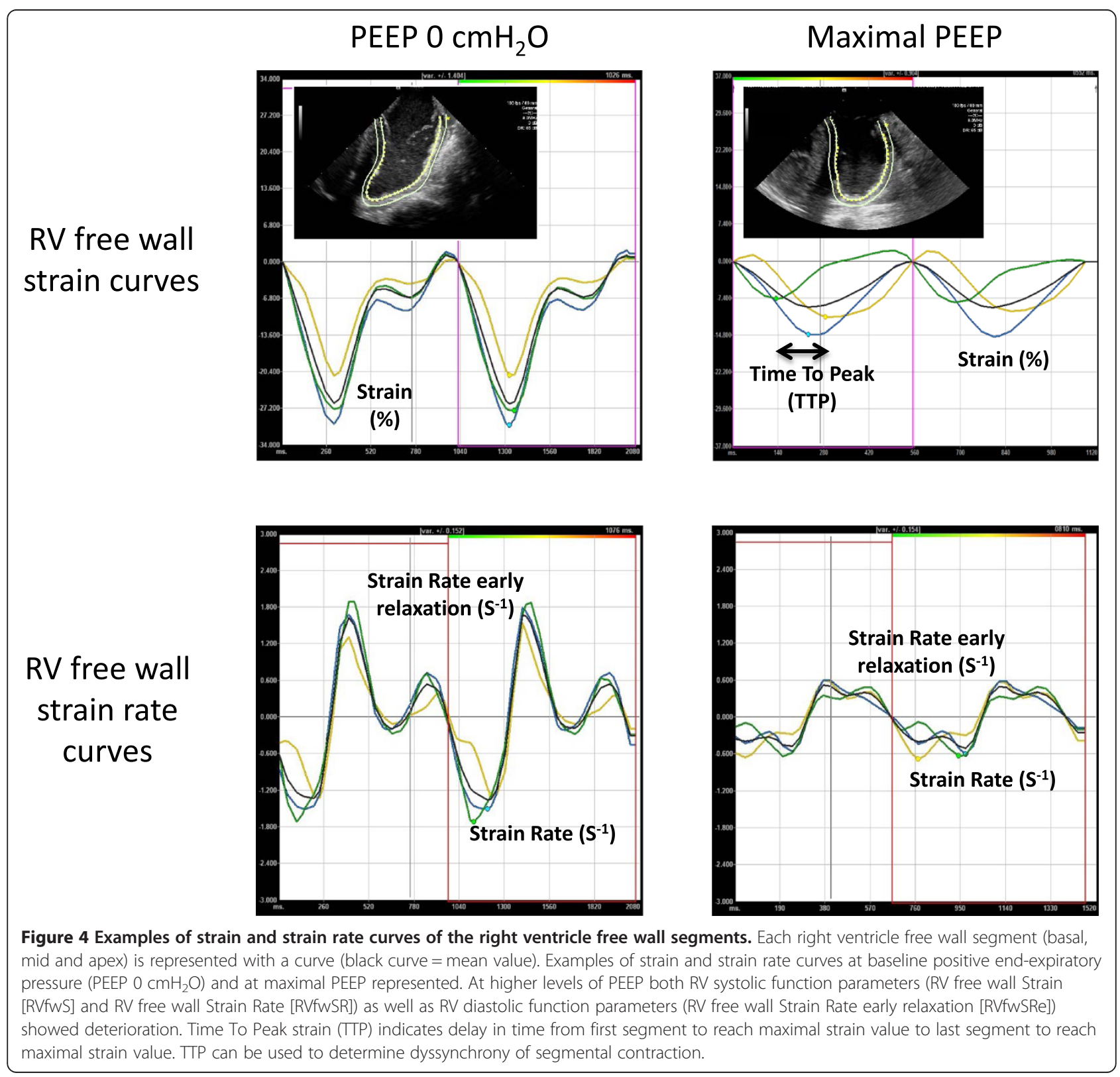


deviations was added to this to obtain the $95^{\text {th }}$ percentile value. We then assessed the TTP delay at the highest PEEP level that was reached for each pig. Dyssynchrony was considered if the TTP delay was above the PEEP 0 $\mathrm{cmH}_{2} \mathrm{O} 95^{\text {th }}$ percentile value.

Statistical analysis was performed with JMP version 10 (SAS Institute Inc., North Carolina). Continuous variables are expressed as mean $+/$ - standard deviation (SD) or median with IQR. Repeated measure analysis at various PEEP levels was done with the one-way ANOVA. If a significant difference was found, post-hoc comparisons between the individual PEEP levels was done using Tukey HSD test with Bonferroni correction for p-value. All probability values are 2 -sided and a value of $\leq 0.05$ was considered significant, except for multiple comparisons between the different PEEP levels where, according to Bonferroni correction, $\mathrm{p}<0.002$ was considered significant.

\section{Results}

Physiological data are presented in Table 1 . In summary: with escalating levels of PEEP there was a trend of a fall in MAP and a rise in mean pulmonary artery pressure (MPAP), however only with MAP were significant differences seen between individual PEEP levels: at $15 \mathrm{cmH}_{2} \mathrm{O}$ and higher vs baseline PEEP $0 \mathrm{cmH}_{2} \mathrm{O}$ (see Figure 5). Significant hypotension, defined as a decrease in MAP of $20 \mathrm{mmHg}$, occurred at approximately $15 \mathrm{cmH}_{2} \mathrm{O}$ PEEP. Higher inspired oxygen levels were required with higher levels of PEEP to maintain oxygen saturation. The step-wise PEEP maneuver was terminated before a PEEP level of $30 \mathrm{cmH}_{2} \mathrm{O}$ in four pigs due to lifethreatening hypotension and bradycardia.

Results of STE analysis (see Figure 6) and conventional echocardiographic analysis of RV function are shown in Table 2. RV systolic function assessed by FAC as well as RVfwS and RVfwSR showed a clear trend towards deterioration with escalating levels of PEEP. RV diastolic function assessed by RVfwSRe also showed a deteriorating trend with escalating PEEP levels. Comparing RVfwS, RVfwSR and RVfwSRe values at different PEEP levels showed a significant difference $(\mathrm{p}<0.002$ as per the Bonferroni correction) at PEEP $0 \mathrm{cmH}_{2} \mathrm{O}$ vs PEEP 10 $\mathrm{CmH}_{2} \mathrm{O}$ and above. In addition RVfwS showed a difference at PEEP $5 \mathrm{cmH}_{2} \mathrm{O}$ vs $15 \mathrm{cmH}_{2} \mathrm{O}$ and above,
RVfwSR and RVfwSRe at PEEP $5 \mathrm{cmH}_{2} \mathrm{O}$ vs $20 \mathrm{cmH}_{2} \mathrm{O}$ and above. RVfwSRe also showed a difference at PEEP $10 \mathrm{cmH}_{2} \mathrm{O}$ vs $25 \mathrm{cmH}_{2} \mathrm{O}$ and above. FAC only showed a significant difference at PEEP $0 \mathrm{cmH}_{2} \mathrm{O}$ vs PEEP 20 $\mathrm{cmH}_{2} \mathrm{O}$ and above as well as PEEP $5 \mathrm{cmH}_{2} \mathrm{O}$ vs 20 $\mathrm{cmH}_{2} \mathrm{O}$ and above. $\mathrm{RVfwS}$ decreased to greater extent compared to baseline level, earlier in the step-wise PEEP escalation process (see Figure 7) and significantly decreased before there was a clinical relevant decrease in MAP.

Analysis of the individual RV free wall segments showed a similar trend of deterioration across segments with escalating PEEP levels (see Table 3). The apical segment did show a significant deterioration at PEEP $10 \mathrm{cmH}_{2} \mathrm{O}$ and above, whereas the basal and mid segments showed a significant deterioration from PEEP $15 \mathrm{cmH}_{2} \mathrm{O}$ upwards. Comparing TTP delay values at PEEP $0 \mathrm{cmH}_{2} \mathrm{O}$ vs final PEEP values, $30 \%$ of pigs had dyssynchrony of the RV free wall at the highest PEEP level reached, defined as a delay of $>108 \mathrm{msec}$ (as determined by mean TTP at PEEP 0 $\mathrm{cmH}_{2} \mathrm{O}+95 \%$ percentile value) between the earliest contracting segment vs the latest contracting segment.

\section{Measurement variability}

Blinded interrater variability for STE analysis was assessed by S.G. on a randomly selected pig at all PEEP levels. Bland-Altman analysis demonstrated good intraobserver and interobserver agreement. The interobserver and intraobserver mean difference ( \pm standard error) were respectively: RVfwS $-1( \pm 0.5)$ and $-0.6( \pm 0.6)$; RVfwSR -0.1 $( \pm 0.1)$ and $-0.1( \pm 0.1) ; \operatorname{RVfwSRe} 0.1( \pm 0.1)$ and $0.1( \pm 0.1)$.

\section{Discussion}

Our study demonstrates a clear trend of reduction in RV function with escalating PEEP levels assessed with a conventional echocardiography method, FAC, and with Speckle Tracking Echocardiography (STE), a novel echocardiography technique. Both RV systolic function parameters measured by STE (RVfwS and RVfwSR) and the diastolic function parameter (RVfwSRe) reduced with elevated PEEP levels. A drop of $20 \mathrm{mmHg}$ in the MAP was considered a clinically relevant end-point and this occurred at approximately PEEP $15 \mathrm{cmH}_{2} \mathrm{O}$. FAC only showed a significant deterioration at PEEP level of $20 \mathrm{cmH}_{2} \mathrm{O}$.

Table 1 Physiological data (values expressed as mean \pm SD)

\begin{tabular}{llllllll}
\hline PEEP $\left(\mathbf{c m H}_{\mathbf{2}} \mathbf{O}\right)$ & PEEP 0 & PEEP 5 & PEEP 10 & PEEP 15 & PEEP 20 & PEEP 25 & PEEP 30 \\
\hline Heart rate $(\mathrm{bpm})$ & $99 \pm 13$ & $104 \pm 18$ & $113 \pm 26$ & $113 \pm 27$ & $109 \pm 30$ & $83 \pm 39$ & $112 \pm 36$ \\
Saturation (\%) & $96 \pm 4$ & $97 \pm 4$ & $91 \pm 7$ & $78 \pm 16$ & $73 \pm 29$ & $90 \pm 15$ & $74 \pm 40$ \\
Fraction inspired oxygen $\left(\mathrm{FiO}_{2}\right)$ & $0.4 \pm 0.1$ & $0.4 \pm 0.1$ & $0.5 \pm 0.1$ & $0.8 \pm 0.2$ & $0.8 \pm 0.2$ & $0.8 \pm 0.2$ & $0.8 \pm 0.2$ \\
Mean Arterial Pressure $(\mathrm{mmHg})$ & $86 \pm 18$ & $73 \pm 15$ & $70 \pm 21$ & $48 \pm 11^{*}$ & $43 \pm 19^{*}$ & $38 \pm 15^{*}$ & $39 \pm 15^{*}$ \\
Mean Pulmonary Artery Pressure $(\mathrm{mmHg})$ & $18 \pm 4$ & $19 \pm 5$ & $21 \pm 6$ & $21 \pm 5$ & $24 \pm 5$ & $27 \pm 5$ & $30 \pm 7$ \\
\hline
\end{tabular}

*indicates significant difference $(\mathrm{p}<0.002)$ compared with PEEP $0 \mathrm{cmH}_{2} \mathrm{O}$. 

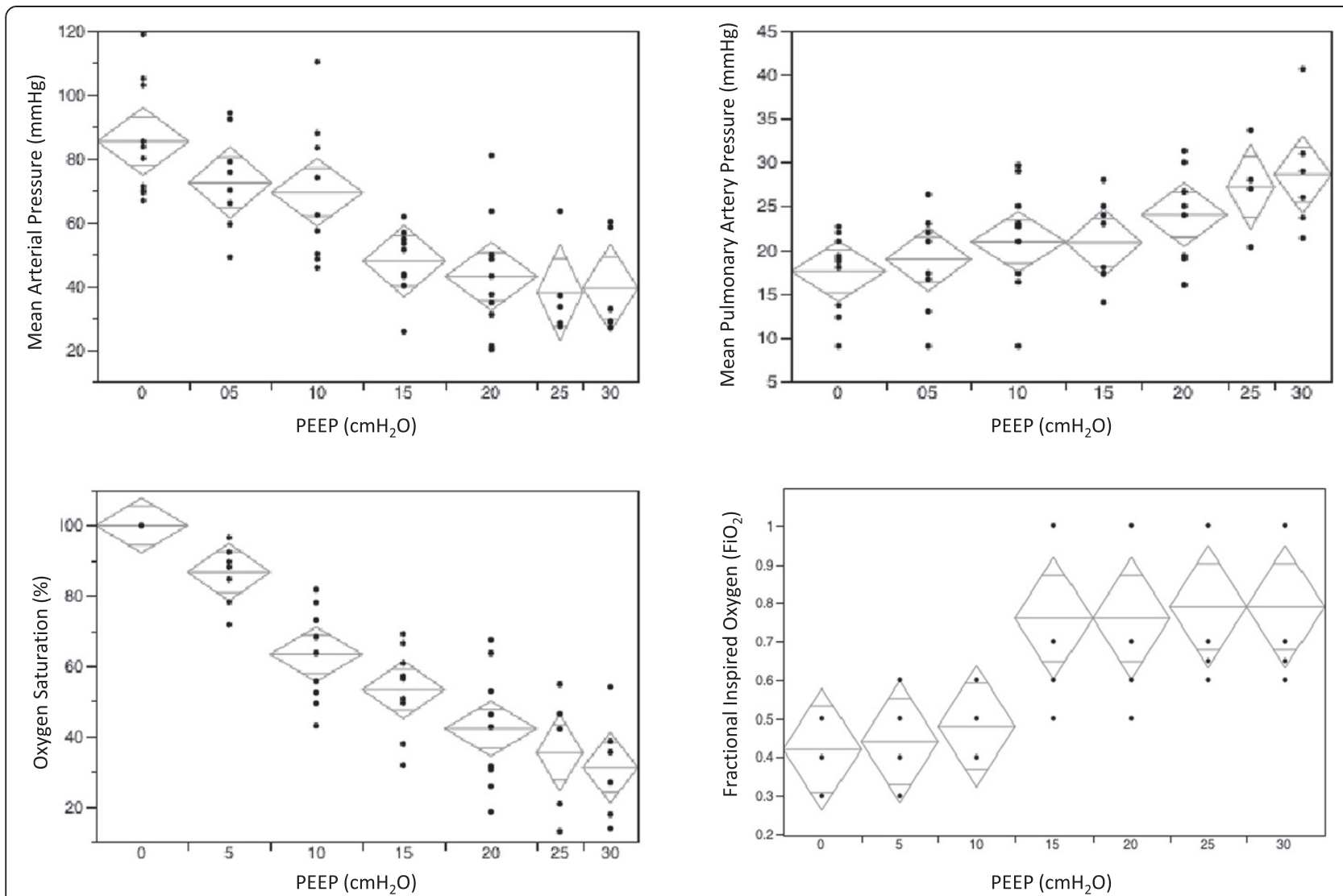

Figure $\mathbf{5}$ Change in physiological parameters with escalating PEEP levels. Mean (+/-95\% confidence limits). Mean arterial pressure, mean pulmonary artery pressure, oxygen saturation and fractional inspired oxygen vs PEEP.

However STE values of systolic function (RVfwS and RVfwSR) and diastolic function (RVfwSRe) showed significant deterioration earlier in the PEEP escalation process: at PEEP $10 \mathrm{cmH}_{2} \mathrm{O}$. No significant difference was seen between either systolic or diastolic parameters at higher PEEP levels suggesting a plateau effect in the degree of RV functional deterioration. Our findings suggest that RV dysfunction induced by PEEP may be identified earlier and with increased sensitivity with STE than by FAC. The effect of PEEP on RV strain has been demonstrated in a study in critically ill patients undergoing a recruitment maneuver [19] indicating the feasibility of this technique in the ICU population.

Echocardiography is an important means of recognizing RV dysfunction induced by mechanical ventilation [6]. Sonographic imaging of the RV can be challenging due to its shape and position and conventional echocardiographic assessment methods are limited by angle-dependence, translational error and often a qualitative approach to analysis. STE is a relatively novel, angle-independent ultrasound imaging technique, which follow groups of grey-scale pixels which create the image of the myocardium (known as 'kernels') and tracks their degree of deformation (strain) and rate of deformation (strain rate) as a surrogate for systolic function [7]. Strain is the most commonly utilized STE value clinically, however animal studies have suggested that strain rate may be a more robust measure of myocardial contractility that is less influence by changes in cardiac load and structure and strain may be influenced in particular by afterload [20]. In our study both RVfwS and RVfwSR were both influenced by PEEP to a similar extent. The initial rate of kernels returning to their end-diastolic position (strain rate early relaxation or RVfwSRe) is a surrogate for diastolic function in much the same way as the e' value with Tissue Doppler Imaging. Although this has not been validated as a clinical reference value at this stage, a small number of animal and clinical studies have shown SRe can identifiy ischaemic areas and viable myocardium in studies of coronary artery disease [21] where diastolic as well as systolic dysfunction occurs.

Unlike the LV, which contracts in all planes (longitudinally, radially, circumferentially with twist and torsion [22]) the RV contracts predominantly in the longitudinal direction due to the dominance of longitudinal muscle fibers in the RV free wall [23]. This places RV free wall strain, which assesses motion in the longitudinal direction, as a sensitive, quantifiable and importantly a feasible tool for assessing RV function non-invasively. Indeed RV free 


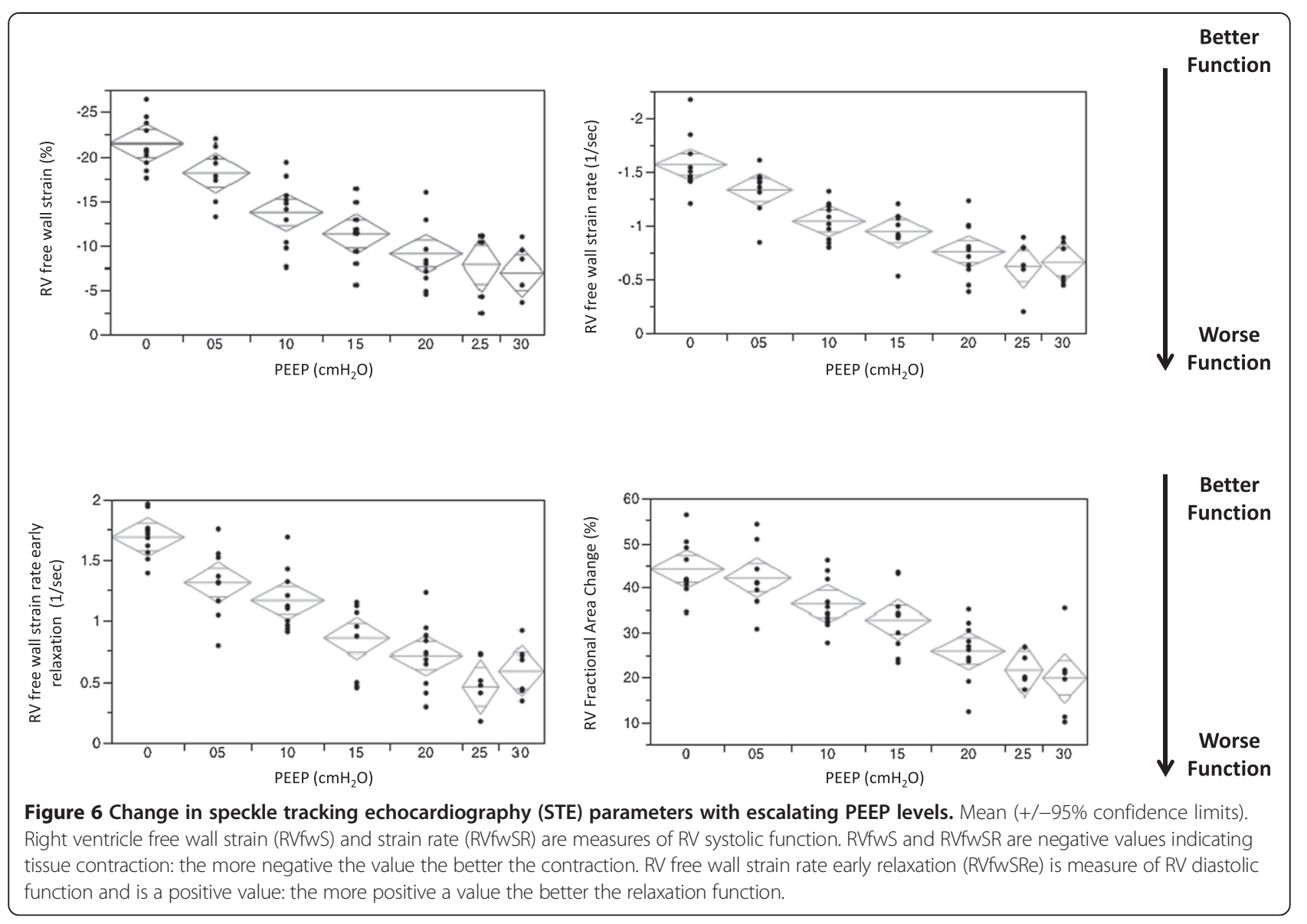

wall strain has been investigated in pulmonary hypertension cohorts and trumps all other echocardiographic methods in predicting both symptom progression and mortality $[10,13]$.

PEEP is an integral part of mechanical ventilation particularly in the critically ill patient with acute lung injury or ARDS. Counteracting alveolar cycling, collapse, derecruitment and to maintain functional residual capacity PEEP aims to reduce hypoxaemia and ventilator-induced lung injury [16]. High levels of PEEP are often recommended in severe ARDS [24] and can affect biventricular function in a variety of complex methods. The exact physiological effects of PEEP on haemodynamics are not entirely elucidated, however RV dysfunction and reduced cardiac output are of serious concern, with cor pulmonale reported in 20-25\% of patients with ARDS [2,25] and is associated with significantly higher mortality [1]. The effect of PEEP on the right ventricle depends on the changes in lung volumes and intrathoracic pressure as well as the underlying pathological state and the physiological response of the pulmonary vasculature [17]. PEEP is reported to predominantly affect $\mathrm{RV}$ afterload resulting in

Table 2 Conventional echocardiography and speckle tracking echocardiography data (values expressed as mean \pm SD)

\begin{tabular}{llllllll}
\hline PEEP $\left(\mathbf{c m H}_{\mathbf{2}} \mathbf{O}\right)$ & PEEP 0 & PEEP 5 & PEEP 10 & PEEP 15 & PEEP 20 & PEEP 25 & PEEP 30 \\
\hline RV EDA $\left(\mathrm{cm}^{2}\right)$ & $11 \pm 3$ & $11 \pm 2$ & $11 \pm 1$ & $10 \pm 2$ & $10 \pm 1$ & $13 \pm 2$ & $13 \pm 1$ \\
RV FAC (\%) & $44 \pm 6$ & $42 \pm 7$ & $36 \pm 6$ & $32 \pm 7$ & $26 \pm 7^{* \mathscr{H}}$ & $22 \pm 4^{* \mathscr{H}}$ & $20 \pm 9^{* \mathscr{H}}$ \\
RVfwS (\%) & $-21.5 \pm 3$ & $-18.2 \pm 3$ & $-13.7 \pm 4^{*}$ & $-11.3 \pm 3^{* \mathscr{H}}$ & $-9.0 \pm 4^{* \mathscr{H}}$ & $-7.8 \pm 4^{* \mathscr{H}}$ & $-6.9 \pm 3^{* \mathscr{H}}$ \\
RVfwSR $\left(^{-1}\right)$ & $-1.6 \pm 0.3$ & $-1.3 \pm 0.2$ & $-1.0 \pm 0.2^{*}$ & $-0.9 \pm 0.2^{*}$ & $-0.8 \pm 0.3^{* \mathscr{H}}$ & $-0.6 \pm 0.3^{* \mathscr{H}}$ & $-0.7 \pm 0.2^{* \mathscr{H}}$ \\
RVfwSRe $\left(^{-1}\right)$ & $1.7 \pm 0.2$ & $1.3 \pm 0.3$ & $1.2 \pm 0.3^{*}$ & $0.9 \pm 0.3^{*}$ & $0.7 \pm 0.3^{* \mathscr{H}}$ & $0.5 \pm 0.2^{* \mathscr{H} \xi}$ & $0.6 \pm 0.2^{* \mathscr{H} \S}$ \\
\hline
\end{tabular}

RV: right ventricle; EDA: end-diastolic area; FAC: fractional area change; RVfwS: right ventricle free wall longitudinal strain; RVfwSR: right ventricle free wall longitudinal free wall strain rate; RVfwSRe: right ventricle free wall longitudinal early strain rate relaxation. NB: RVfwS and RVfwSR are systolic function parameters and are expressed as negative values: the less negative a value, the better the function. RVfwSRe is a diastolic function parameter and expressed as a positive value: the more positive, the better the relaxation function. *indicates significant difference $(\mathrm{p}<0.002)$ compared with PEEP $0 \mathrm{cmH}_{2} \mathrm{O}$. ${ }^{{ }^{\circ}}$ indicates significant difference compared with $\mathrm{PEEP} 5 \mathrm{cmH} \mathrm{H}_{2} \mathrm{O}$.

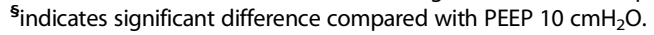




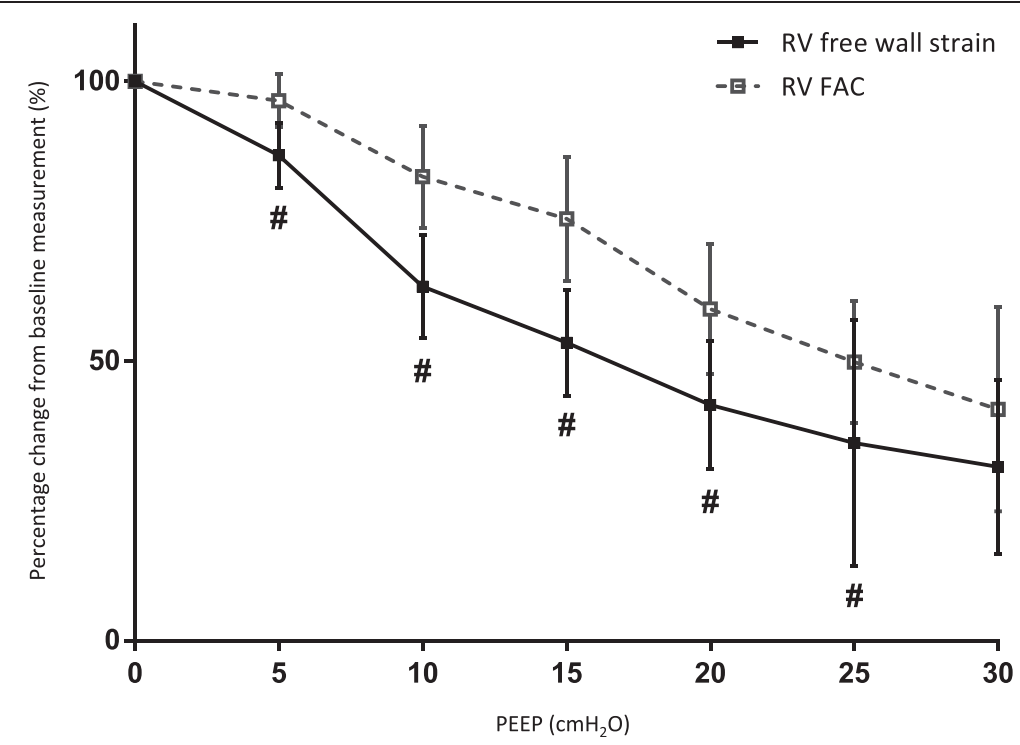

Figure 7 Percentage change from baseline level in right ventricle free wall strain (RVfwS) and fractional area change (FAC). RVfwS reduces to a greater extent than FAC and earlier in the PEEP escalating process.

a reduced RV stroke volume through increased RV outflow impedance in ARDS patients [26,27] and there are reports of increased RV end-diastolic area [25]. This has led to the concept of a 'RV protection' approach to mechanical ventilation in ARDS patients, which limits PEEP and avoids hypercapnic acidosis [28]. STE potentially may provide a method for identifying RV failure induced by PEEP ahead of conventional methods of RV function assessment. This has the potential to allow the physician to direct therapy earlier at protecting the RV [25].

\section{Limitations}

STE, as with conventional echocardiography, is limited by adequate image quality. We utilized ICE in order to maximize the imaging quality of the RV free wall as neither transthoracic or transoesophageal echocardiography could reliably be performed to provide sufficient image quality of the RV free wall for STE analysis. This relates to the mediastinal anatomy of the pig model. The use of STE with ICE has not been validated, however STE analysis is angle-independent, was feasible and each pig acted as its own control. The ultrasound equipment is comparable and the only difference is the transducer. Our data should be translatable to echocardiography images acquire by other transducers. However, the need to use ICE prevented many of the standard echocardiography measures of RV function such as TAPSE and Sm by Tissue Doppler Imaging as these values are angle dependent and require apical imaging. FAC was the most plausible method to assess RV function as recommended by ASE guidelines [29]. Tachycardia can also impair the software's ability to accurately track the speckles of the image, and heart rates greater than 100 were frequently observed particularly during the escalating PEEP process. We performed the step-wise PEEP escalation process in pigs with healthy lungs, pigs with diseased lung and reduced compliance may affect results.

\section{Conclusion}

RV dysfunction in the critically ill is known to be associated with poor outcomes and can be induced by mechanical ventilation and PEEP therapy. Speckle tracking echocardiography is a quantifiable, sensitive and feasible angleindependent method for detecting RV dysfunction induced

Table 3 Right ventricle free wall strain segmental data (values are expressed as mean \pm SD)

\begin{tabular}{|c|c|c|c|c|c|c|c|c|}
\hline PEEP $(\mathrm{cmH} 2 \mathrm{O})$ & PEEP 0 & PEEP 5 & PEEP 10 & PEEP 15 & PEEP 20 & PEEP 25 & PEEP 30 & $\begin{array}{l}\text { Mean difference from } \\
\text { PEEP } 0 \text { to final PEEP }\end{array}$ \\
\hline Basal segment S(\%) & $-19.2 \pm 4$ & $-15.8 \pm 3$ & $-12.3 \pm 5$ & $-8 \pm 5^{*}$ & $-9 \pm 1^{*}$ & $-7 \pm 4^{*}$ & $-6 \pm 4^{*}$ & $14.0 \pm 1$ \\
\hline Mid segment S(\%) & $-23.4 \pm 4$ & $-19.7 \pm 5$ & $-15.1 \pm 5$ & $-11.2 \pm 4^{*}$ & $-8.6 \pm 5^{* \mathscr{H}}$ & $-9.3 \pm 6^{*}$ & $-8.7 \pm 5^{* \mathscr{H}}$ & $15.5 \pm 2$ \\
\hline Apical segment S(\%) & $-22 \pm 4$ & $-19 \pm 4$ & $-12.7 \pm 5^{*}$ & $-13.3 \pm 3^{*}$ & $-9.3 \pm 3^{* 26}$ & $-7.3 \pm 3 * 26$ & $-7 \pm 3^{* 28}$ & $15.2 \pm 1$ \\
\hline TTP delay (msec) & $50 \pm 29$ & $78 \pm 58$ & $72 \pm 28$ & $69 \pm 43$ & $113 \pm 61$ & $118 \pm 133$ & $86 \pm 70$ & $71.3 \pm 31$ \\
\hline
\end{tabular}

S: strain; TTP delay: time to peak strain delay between segmental values. ${ }^{*}$ indicates significant difference $(p<0.002)$ compared with PEEP $0 \mathrm{cmH}_{2} \mathrm{O}$. ${ }^{\text {If }}$ indicates significant difference compared with PEEP $5 \mathrm{cmH}_{2} \mathrm{O}$. 
by escalating PEEP levels, and may display dysfunction ahead of conventional echocardiographic methods of assessment. The STE software is available on most current high-end machines, and is becoming increasingly available in intensive care units world-wide. Further studies in the ICU population, particularly with acute lung injury and ARDS, using transthoracic imaging are warranted.

\section{Abbreviations}

ARDS: Acute respiratory distress syndrome; EDA: End-diastolic area; FAC: Fractional area change; FiO2: Fraction of inspired oxygen; ICE: Intracardiac echocardiography; IQR: Interquartile range; MAP: Mean arterial pressure; MPAP: Mean pulmonary artery pressure; PEEP: Positive end expiratory pressure; RV: Right ventricle; RVfwS: Right ventricle free wall longitudinal strain; RVfwSR: Right ventricle free wall longitudinal free wall strain rate; RVfwSRe: Right ventricle free wall longitudinal early strain rate relaxation; S: Strain; SD: Standard deviation; STE: Speckle tracking echocardiography; TTP delay: Time to peak strain delay between segmental values.

\section{Competing interests}

The authors declare that they have no competing interests.

\section{Authors' contributions}

SO, AB, PS, GK and JO contributed to study conception and design, preparation and revision of the manuscript and take responsibility for the integrity and accuracy of the data and analysis. SO, SG, AB and PS contributed to data acquisition. SO, AB, SG, GK and JO contributed to analysis and interpretation of the data and revision of the manuscript. All authors read and approved the final manuscript.

\section{Acknowledgements}

This study was supported by Grainger Foundation, Florida Heart Research Institute, Marriott Heart Disease Research Program, and Center for Regenerative Medicine, Mayo Clinic (USA). Thanks to Associate Professor Stephen Huang (Nepean Hospital, Sydney) for help with statistical analysis in the manuscript revision process.

Received: 4 February 2015 Accepted: 24 March 2015

Published online: 11 April 2015

\section{References}

1. Boissier F, Katsahian S, Razazi K, Thille AW, Roche-Campo F, Leon R, et al. Prevalence and prognosis of cor pulmonale during protective ventilation for acute respiratory distress syndrome. Intensive Care Med. 2013;39:1725-33.

2. Dessap AM, Boissier F, Leon R, Carreira S, Campo FR, Lemaire F, et al. Prevalence and prognosis of shunting across patent foramen ovale during acute respiratory distress syndrome*. Crit Care Med. 2010;38:1786-92.

3. Lahm T, McCaslin C, Wozniak T, Ghumman W, Fadl Y, Obeidat O, et al. Medical and surgical treatment of acute right ventricular failure. J Am Coll Cardiol. 2010;56:1435-46.

4. Bouferrache K, Vieillard-Baron A. Acute respiratory distress syndrome, mechanical ventilation, and right ventricular function. Curr Opin Crit Care. 2011;17:30-5.

5. Vieillard-Baron A. Assessment of right ventricular function. Curr Opin Crit Care. 2009;15:254-60.

6. Greyson CR. Right heart failure in the intensive care unit. Curr Opin Crit Care. 2012;18:424-31.

7. Huang SJ, Orde S. From speckle tracking echocardiography to torsion. Curr Opin Crit Care. 2013;19:250-7.

8. Orde SR, Pulido JN, Masaki M, Gillespie S, Spoon JN, Kane GC, et al. Outcome prediction in sepsis: speckle tracking echocardiography based assessment of myocardial function. Crit Care. 2014;18:R149.

9. Guendouz S, Rappeneau S, Nahum J, Dubois-Randé J-L, Gueret P, Monin J-L, et al. Prognostic significance and normal values of $2 \mathrm{D}$ strain to assess right ventricular systolic function in chronic heart failure. Circ J. 2012;76:127-36.

10. Hardegree EL, Sachdev A, Villarraga HR, Frantz RP, McGoon MD, Kushwaha SS, et al. Role of serial quantitative assessment of right ventricular function by strain in pulmonary arterial hypertension. Am J Cardiol. 2013;111:143-8.
11. Haeck M, Scherptong R, Marsan N, Holman E, Schalij M, Bax J, et al. Prognostic value of right ventricular longitudinal peak systolic strain in patients with pulmonary hypertension. Circ Cardiovasc Imaging. 2012;5:628-36.

12. Sachdev A, Villarraga HR, Frantz RP, McGoon MD, Hsiao J-F, Maalouf JF, et al. Right ventricular strain for prediction of survival in patients with pulmonary arterial hypertension. Chest. 2011;139:1299-309.

13. Fine NM, Chen L, Bastiansen PM, Frantz RP, Pellikka PA, Oh JK, et al. Outcome prediction by quantitative right ventricular function assessment in 575 subjects evaluated for pulmonary hypertension. Circ Cardiovasc Imaging. 2013;6:711-21.

14. Pinsky MR. Cardiovascular issues in respiratory care. Chest. 2005;128:592S-7S.

15. Gattinoni L, Carlesso E, Brazzi L, Caironi P. Positive end-expiratory pressure. Curr Opin Crit Care. 2010;16:39-44.

16. Dreyfuss D, Saumon G. Ventilator-induced lung injury: lessons from experimental studies. Am J Respir Crit Care Med. 1998;157:294-323.

17. Luecke T, Pelosi P. Clinical review: positive end-expiratory pressure and cardiac output. Crit Care. 2005;9:607-21.

18. Yu C-M, Lin H, Zhang Q, Sanderson JE. High prevalence of left ventricular systolic and diastolic asynchrony in patients with congestive heart failure and normal QRS duration. Heart. 2003;89:54-60.

19. Franchi F, Faltoni A, Cameli M, Muzzi L, Lisi M, Cubattoli L, et al. Influence of positive end-expiratory pressure on myocardial strain assessed by speckle tracking echocardiography in mechanically ventilated patients. Biomed Res Int. 2013;2013:918548.

20. Ferferieva V, Van den Bergh A, Claus P, Jasaityte R, Veulemans P, Pellens M, et al. The relative value of strain and strain rate for defining intrinsic myocardial function. AJP Heart Circ Physiol. 2011;302:H188-95.

21. Oh JK, Park S-J, Nagueh SF. Established and novel clinical applications of diastolic function assessment by echocardiography. Circ Cardiovasc Imaging. 2011;:4:444-55

22. Sanderson JE. Left and right ventricular long-axis function and prognosis. Heart (British Cardiac Society). 2008;94:262-3.

23. Rushmer R, Crystal $D$. Changes in configuration of the ventricular chambers during the cardiac cycle. Circulation. 1951;4:211-8.

24. Rouby J-J, Lu Q, Goldstein I. Selecting the right level of positive end-expiratory pressure in patients with acute respiratory distress syndrome. Am J Respir Crit Care Med. 2002;165:1182-6.

25. Vieillard-Baron A, Schmitt JM, Augarde R, Fellahi JL, Prin S, Page B, et al. Acute cor pulmonale in acute respiratory distress syndrome submitted to protective ventilation: incidence, clinical implications, and prognosis. Crit Care Med. 2001;29:1551-5.

26. Vieillard-Baron A, Loubieres Y, Schmitt JM, Page B, Dubourg O, Jardin F. Cyclic changes in right ventricular output impedance during mechanical ventilation. J Appl Physiol. 1999:87:1644-50.

27. Schmitt JM, Vieillard-Baron A, Augarde R, Prin S, Page B, Jardin F. Positive end-expiratory pressure titration in acute respiratory distress syndrome patients: impact on right ventricular outflow impedance evaluated by pulmonary artery Doppler flow velocity measurements. Crit Care Med. 2001;29:1154-8.

28. Vieillard-Baron A, Jardin F. Right level of positive end-expiratory pressure in acute respiratory distress syndrome. Am J Respir Crit Care Med. 2003:167:1576. -author reply 1576-7.

29. Rudski LG, Lai WW, Afilalo J, Hua L, Handschumacher MD, Chandrasekaran K et al. Guidelines for the echocardiographic assessment of the right heart in adults: a report from the American Society of Echocardiography endorsed by the European Association of Echocardiography, a registered branch of the European Society of Cardiology, and the Canadian Society of Echocardiography. J Am Soc Echocardiogr. 2010;23:685-713. quiz 786-8. 